\title{
EDITORIAL
}

\section{Current and future challenges in otorhinolaryngology}

\author{
Raluca Enache, MD, PhD' \\ ${ }^{1}$ ENT Sarafoleanu Medical Clinic, Bucharest, Romania
}

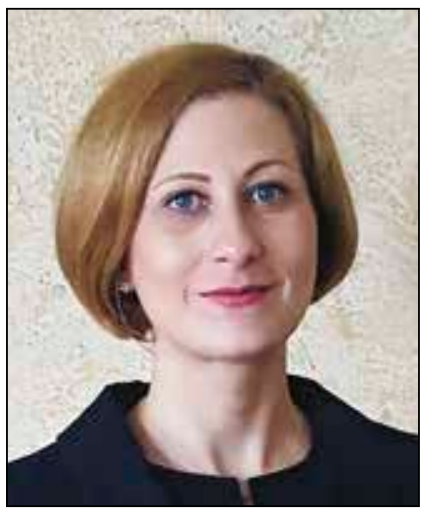

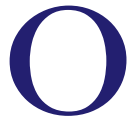
ver time, medicine has been in continuous development. The challenges and innovations in medicine are numerous, rapid, and can be difficult to assimilate and manage in a timely manner without rigorous training. All these challenges supported by scientific research are more and more, with a sequentiality almost impossible to mention: new treatment methods for rare and incurable diseases (cancer, diabetes, obesity), stem cell treatments, organ transplantation improvement, robotic surgery, nanomedicine and nanotherapies, genetics and gene therapies.

Under these circumstances, in Otorhinolaryngology also, the challenges are important, rapid and must be well managed. An important share will be held by outpatient medicine that, through international statistics, has proven that it can satisfy up to $75 \%$ of the specialty needs ${ }^{1}$, which leads to a low number of beds and savings that can be invested in technical equipment and patient satisfaction. Given the development of research and technology, otorhinolaryngology (ENT) is increasingly involved in the borderline pathology (neurosurgery, cervical and vascular surgery, otoneurology, rhino-neurology, etc.), which requires translationality, that is, a multidisciplinary team. In this context, monoprofile hospitals are no longer topical. Current research in ENT focuses on areas such as: head and neck cancers, inflammatory rhinosinusal pathology, vestibular and auditory system, olfactory pathology, surgical techniques in the ENT field.

Treatment of head and neck cancer. Squamous cell carcinomas have the highest incidence among head and neck cancers. Until recently, the decision regarding the therapeutic conduct depended primar- ily on the TNM staging and on the local evolution of the tumor. In recent years, starting from the active involvement of HPV (human papillomavirus) and smoking in the occurrence of these cancers, research has focused on the molecular biology and genomics of head and neck cancers, with important implications in treating this pathology. An important role is played by the Cancer Genome Atlas (TCGA), consortium from the National Cancer Institute Organization structure, which after 12 years of research actively contributes to establishing the importance of genomics in the treatment of cancer. What did TCGA researchers discover? The molecular difference between head and neck cancers associated with HPV infection and / or smoking and those not associated with it ${ }^{2}$. HPV-positive tumors are associated with mutations in genes that are involved either in regulating the cell cycle or in the body's immune response, or in cell proliferation, migration and survival. HPV-negative tumors are associated with chromosomal changes, the result being the inhibition of cellular apoptosis. Tumors developed in smokers are associated with mutations in the p53 gene, leading to the reduction or absence of suppressor proteins of cancer cells with secondary tumor proliferation. All these findings have led to the study of new antibody-based treatments to counteract the immune resistance of tumor cells and increase the effectiveness of these therapies.

Inflammatory rhinosinusal pathology. Clinically, there are two important entities of rhinosinusitis - chronic rhinosinusitis without nasal polyps (CRSsNP) and chronic rhinosinusitis with nasal polyps (CRSwNP) - which differ clinically, but also molecularly and biologically. If Th cytokines and a neutrophil-rich inflammatory infiltrate are in- 
volved in CRSsNP, CRSwNP is characterized by an inflammatory process mediated by Th2 cytokines, elevated IgE levels, interleukins (IL-4, IL-5, IL-13), vascular endothelial growth factor (VEGF), intercellular adhesion molecule 1 (ICAM-1), with a role in activating eosinophils, inhibition of eosinophil apoptosis and increased eosinophilic infiltrate in the nasal mucosa ${ }^{3}$. Specialty guidelines effectively systematize the drug treatment of CRS (corticosteroids, antibiotic, irrigation with saline solutions, antileukotrienes, aspirin desensitization therapy).

In recent years, research has been conducted on the efficacy of monoclonal antibodies in the treatment of CRSwNP, an immunotherapy that uses monoclonal antibodies that specifically bind to certain target cells or proteins (for example, immunoglobulins, interleukins): Anti-IgE monoclonal antibodies (reduce circulating $\operatorname{IgE}$, tissue eosinophilia, Th2-type cytokine production; decrease the symptom score and the need for drug treatment), Anti-IL-4 / IL-13 monoclonal antibodies (decrease the concentration of eosinophils in the tissue; reduce the volume of nasal polyps; decrease the dose of systemic corticosteroids required; decrease the risk of recurrence); Anti-IL-5 monoclonal antibodies (significantly decrease polypoid volume) $)^{3}$.

Recent studies are targeting new agents that may be effective in treating CRSwNP: anti-TSLP (thymic stromal lymphopoietin), anti-TNF, anti-IL-8, CRTH2 agonists $^{3}$. However, more information is needed.

Auditory and vestibular system. In recent years, new possible treatment methods for sensorineural hearing loss (SNHL) have been investigated, knowing that destroyed cochlear hair cells do not regenerate in humans. It seems that SNHL treatment with umbilical cord blood stem cells (study conducted in Florida, USA) would result in the regeneration of hair cells and supporting cells in the cochlea ${ }^{4}$. The second possible treatment involves EGF (epidermal growth factor) and its role in cochlear cell regeneration. Researchers at the University of Rochester Medical Center and Harvard Medical School's Massachusetts Eye and Ear Infirmary have shown in studies performed on mice both the regeneration of sensory hair cells and their connections with nerve cells ${ }^{5}$. Gene therapy is also part of new ongoing clinical studies and could help improve hearing by increasing intelligibility.

Regarding the vestibular system, the lines of research involve: understanding how age alters the vestibular function, thus influencing mobility and the risk of falling in the elderly with the involvement of genetics; development of new technologies for vestibular evaluation and rehabilitation through the use of the vestibulo-ocular reflex and / or virtual reality. The study of a vestibular implant developed and performed at Johns Hopkins Hospital could be an important help for patients with partial or total vestibular deficiency ${ }^{6}$.

Olfactory pathology. The development of new methods for olfactory and gustatory evaluation, new tests to identify and document the development, regeneration and functioning of chemosensory cells, which further help to develop new and effective treatments (such as gene or stem cell therapies) ${ }^{7}$.

Surgical techniques in the ENT field have also known an important development over time. At present, robotic surgery is the one that knows a progressive evolution ${ }^{8}$. Transoral robotic surgery initially began to be used in the malignant and benign pathology of the oropharyngeal and supraglottic region (tongue base resection, tongue base volumetric reduction, uvulopalatoplasty). Subsequently, laryngeal surgery began to be performed with the help of the robot: supraglottic laryngectomy, the surgery of laryngoceles, cordectomies (stages T1, T2). There are several cases in which total laryngectomy was attempted by robotic surgery, but the results were not as expected. Reconstructions of post-surgical pharyngeal defects, skull base surgery, the transcervical approach in thyroid surgery are still in the study phase.

Clinical and laboratory studies address laryngeal transplantation and selective reinnervation of the transplanted larynx. The results so far have shown that the best candidates would be patients with total laryngectomy for laryngeal trauma, benign or low-grade tumors ${ }^{9}$.

The $3 D$ printer finds its applicability in otology, rhinology, head and neck pathology ${ }^{10}$. In preclinical and surgical education, the printer can help print models for temporal bone dissection, middle ear surgery, ear and rhinosinusal endoscopic surgery, skull base surgery, tracheostomy, or as an anatomical model. 3D reproduction of different anatomical regions depending on the appearance of the preoperative CT can help the surgeon plan the surgery. Implantable prostheses or tissue can be developed (repair of the superior semicircular canal dehiscence; recovery of bone loss in case of a craniofacial fibrous dysplasia; mandibular reconstructions).

The discovery of new anatomical regions is part of the natural evolution of medicine. A team of researchers from the Netherlands, led by Dr. Matthijs H. Valstar ${ }^{11}$, reported the discovery of a new pair of salivary glands in the nasopharynx, using positron emission computed tomography (PET/ $\mathrm{CT}$ ) with radiolabelled ligands for the membrane- 
specific antigen in prostate or para-urethral cancer. The pathological examination of the corpse revealed glandular tissue and secretory ducts.

Medicine is in a continuous transformation, progress being the only method by which we can discover new methods for evaluating the human body and new methods for treating human pathology.

\section{REFERENCES}

1. de Gabory L, Sowerby LJ, DelGaudio JM, Al-Hussaini A, Hopkins C, Serrano E. International survey and consensus (ICON) on ambulatory surgery in rhinology. Eur Ann Otorhinolaryngol Head Neck Dis. 2018;135(1S):S49-53.

2. The Cancer Genome Atlas Network., Genome sequencing centre: Broad Institute., Lawrence M., et al. Comprehensive genomic characterization of head and neck squamous cell carcinomas. Nature. 2015;517:576-82. DOI: 10.1038/nature14129.

3. Kariya S, Okano M, Nishizaki. Biological basis and clinical implications of immunological molecules involved in eosinophilic inflammation in allergic ehinitis, chronic ehinosunusitis, and asthma. Advances in Cellular and Molecular Otolaryngology. 2015;3:1. DOI: 10.3402/acmo.v3.26601.0

4. Baumgartner LS, Moore E, Shook D, Messina S, Day MC, Green J, et al. Safety of autologous umbilical cord blood therapy for acquired sensorineural hearing loss in children. J Audiol Otol. 2018;22(4):20922. DOI: $10.7874 /$ jao.2018.00115.

5. Zhang J, Wang Q, Abdul-Aziz D, Mattiacio J, Edge ASB, White PM. ERBB2 signaling drives supporting cell proliferation in vitro and apparent supernumerary hair cell formation in vivo in the neonatal mouse cochlea. Eur J Neurosci. 2018;48(10):3299-316. DOI: 10.1111/ejn.14183.

6. Chow MR, Ayiotis AI, School DP, Gimmon Y, Lane KE, Morris BJ, et al. Posture, gait, quality of life, and hearing with a vestibular implant. N Engl J Med. 2021;384(6):521-32. DOI: 10.1056/NEJMoa2020457.

7. Mainland JD, Barlow LA, Munger SD, Millar SE, Vergara MN, Jiang $\mathrm{P}$, et al. Identifying treatments for taste and smell disorders: gaps and opportunities. Chemical Senses. 2020;45(7):493-502.

8. Byrd JK, Duvvuri U. Curernt trends in robotic surgery for otolaryngology. Curr Otorhinolaryngol Rep. 2013;1(3):153-7. DOI: 10.1007/ s40136-013-0025-6.

9. Sakallioglu O. Laryngeal transplantation. Turk Arch Otorhinolaryngol. 2015;53(3):128-32.

10. Canzi P, Magnetto M, Marconi S, Morbini P, Mauramati S, Aprile F, et al. New frontiers and emerging applications of 3D printing in ENT surgery: a systematic review of the literature. Acta Otorhinolaryngol Ital. 2018;38:286-303.

11. Valstar MH, de Bakker BS, Steenbakkers RJHM, de Jong KH, Smit LA, Klein Nulet TJW, et al. The tubarial salivary glans: A potential new organ at risk for radiotherapy. Radiother Oncol. 2020;S01678140(20)30809-4. DOI: 10.1016/j.radonc.2020.09.034. 